\title{
Wide Angle X-Ray Scattering to Study the Atomic Structure of Polymeric Fibers
}

\author{
Teresa Sibillano, Alberta Terzi, Liberato De Caro®, Massimo Ladisa, Davide Altamura, \\ Anna Moliterni, Rocco Lassandro, Francesco Scattarella, Dritan Siliqi ${ }^{\circ}$ and Cinzia Giannini * \\ Institute of Crystallography-National Research Council (IC-CNR), via Amendola 122/O, 70126 Bari, Italy; \\ teresa.sibillano@ic.cnr.it (T.S.); alberta.terzi@ic.cnr.it (A.T.); liberato.decaro@ic.cnr.it (L.D.C.); \\ massimo.ladisa@ic.cnr.it (M.L.); davide.altamura@ic.cnr.it (D.A.); annagrazia.moliterni@ic.cnr.it (A.M.); \\ rocco.lassandro@ic.cnr.it (R.L.); francesco.scattarella@ic.cnr.it (F.S.); dritan.siliqi@ic.cnr.it (D.S.) \\ * Correspondence: cinzia.giannini@ic.cnr.it
}

Received: 6 March 2020; Accepted: 1 April 2020; Published: 4 April 2020

\begin{abstract}
Natural fibrillar-like macromolecules find applications in several fields, thanks to their peculiar features, and are considered perfect building blocks for natural and artificial functional materials. Indeed, fibrous proteins (such as collagen or fibroin) are commonly used in scaffold fabrication for biomedical applications, due to the high biophysical similarity with the extracellular matrix (ECM) which stimulates tissue regeneration. In the textile industry, cellulose-based fabrics are widely used in place of cotton and viscose, which both have sustainability issues related to their fabrication. With this in mind, the structural characterization of the materials at molecular scale plays a fundamental role in gaining insight into the fiber assembly process. In this work, we report on three fibers of research interest (i.e., type I collagen, silk fibroin extracted from Bombyx mori, and cellulose) to show the power of wide-angle X-ray scattering to characterize both intra- and intermolecular parameters of fibrous polymers. The latest possibilities offered in the X-ray scattering field allow one to study fibers at solid state or dispersed in solutions as well as to perform quantitative scanning X-ray microscopy of tissues entirely or partially made by fibers.
\end{abstract}

Keywords: fiber diffraction; WAXS; X-rays; natural polymers

\section{Introduction}

Over recent years, there has been a growing interest in the development of fibrillar structures containing, as the main fibrous structures, natural (cellulose, collagen, silk fibroin, elastin, chitosan, and alginate) and synthetic (polylactic acid-PLA, poly-lactic-co-glycolic acid-PLGA, polycaprolactone-PCL) polymers, separately or in a blend. Thanks to their tunable specific features, linked to their order at molecular scale/nanoscale, fibrous materials have found applications in several fields. In particular, when fibers possess diameters ranging from 10 to more than $100 \mathrm{~nm}$, i.e., nanofibers, they show a high surface-to-volume ratio, and high sensitivity to external physical and chemical stimuli with respect to the micro fibrous materials [1,2], thus enhancing interaction with a final target. The possibility to tune the fiber size and orientation, the high porous structure, together with the possibility to construct networks with controlled structure and interconnected porosity, making them useful for the development of functional materials applied to several fields, such as tissue engineering [3], water filtration [4], semiconducting materials [5] and energy generation and storage [6]. The choice of the materials for fiber fabrication is strongly related to the final function of the device.

Natural fibrous polymers are often employed for scaffold or drug delivery devices production in biomedical applications [7] or drug/gene delivery devices, because of their biocompatibility and non-toxic biodegradation. It has been demonstrated that fibrillary scaffolds made of natural polymers 
improve cell adhesion, growth and tissue regrowth as they are able to mimic natural biochemical and topographic features of the extracellular matrix (ECM) [8], in particular, superficial roughness, viscoelasticity, and sub- and supra-molecular configuration. Wound dressing and tissue regeneration appear to be the favorite fields of application of fibrous polymers that possess great air permeation, preventing infection and promoting wound healing [9]. Moreover, scaffolds made of oriented fibers are used as "railways" for anisotropic tissue regeneration or scaffold fabrication of bone, skin connectives, muscles and nerves, as they are physical guides that promote tenocyte growth along the axis of the tendon and/or axonal regrowth. Synthetic fibrous devices are developed for tissue engineered constructs, because of their low antigenicity, but also for other manufacturing fields. Fibrous synthetic materials are also employed in the aerospace industry in order to enhance the mechanical reinforcement [10] of the structures. In the textile field, nanostructured fiber-based garments are able to keep body temperature constant, to prevent rain and cold infiltration and to provide comfort thanks to the controlled nanoporosity [11]. Moreover, fibers appear also to be useful enhancers of sensitivity in sensor development [12] and long-term electrolyte storage.

Considering the crystallographic classification of crystalline materials, three major categories can be identified (see Figure 1):

1) single crystals due to the periodic repetition of the same crystal unit cell in three directions of space, forming a quite large single crystalline domain (extension of the domain: from several tenths of microns to a few millimeters), whose data set of diffraction patterns is characterized by a spotted-like diffraction intensity distribution;

2) bundle of fibers, which are crystals ordered along two main directions, the meridional direction along the fiber axis and the equatorial direction perpendicular to it, whose diffraction pattern is made by arcs mainly localized along the meridional and equatorial directions, respectively;

3) polycrystalline materials (or powders), which are made by thousands of small single crystals, oriented in all directions, whose diffraction pattern is marked by concentric rings.

The information which can be extracted by a 2D wide-angle diffraction pattern of single crystal, bundle of fibers and polycrystalline material, respectively, is therefore the fingerprint of the specific material crystallinity class, as the intensity distribution allows us to characterize each crystal in its category without uncertainty.

Single crystal

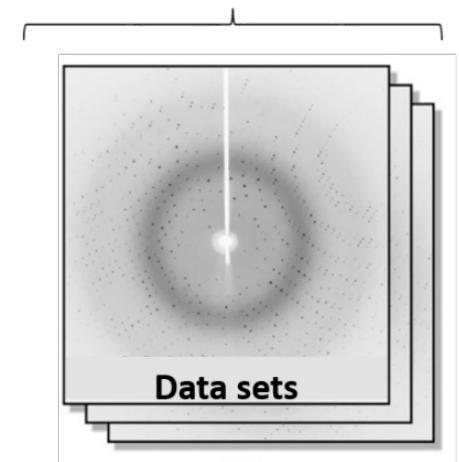

Bundle of fibers

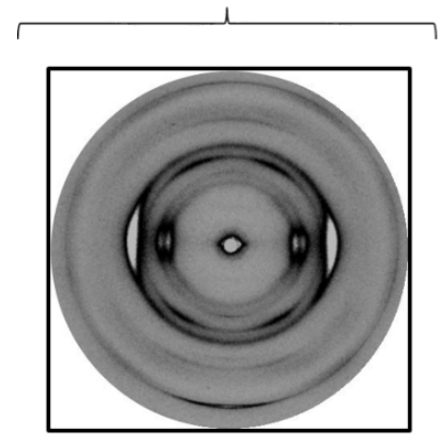

Polycrystals

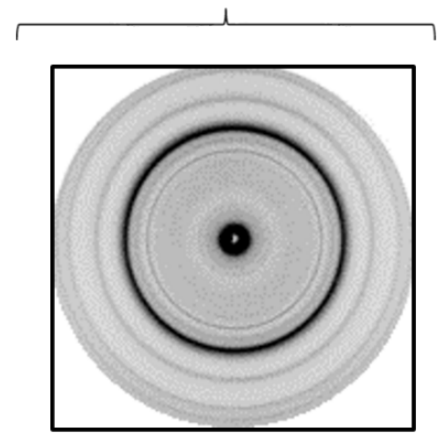

Figure 1. Typical diffraction patterns of single-crystal (left); bundle of fibers (center); poly-crystals (right).

Small- and wide-angle X-ray scattering (SAXS and WAXS) are among the most suitable methods to investigate fibrous macromolecular polymer assemblies to obtain structural details with nanometric or atomic resolution, respectively. The characterization by both SAXS and WAXS, especially to study hierarchical systems, is extremely up-to-date and beneficial to a whole analysis of the structure across the different length scales, as shown by Terzi et al. in collagen type 1 [13]. 
When fibers are embedded in natural tissues or artificial scaffolds, SAXS and WAXS scanning microscopy can be obtained by mapping areas of several $\mathrm{mm}^{2}$ with a focused X-ray beam $[14,15]$. The combination of crystallographic and statistical methods can transform raw SAXS/WAXS datasets into quantitative images, to map the variation of specific structural properties in the explored area. These microscopies have been used to study the organization of the fibers into natural tissues like eyes, teeth, bone, skin connectives, muscles and nerves, as well as in engineered ones [15-17].

In addition, fibers can also be studied in a water solution [18] resembling the condition of fibers dispersed in biological liquids as well as a solution containing an injectable drug.

Information extracted from fiber diffraction/scattering, both in a solid or liquid phase, respectively, can be combined with atomic models to determine or even predict behaviors and features of fiber-based materials. Last but not least, fibers have also been largely studied with small angle neutron scattering (SANS). New instruments such as BEER and HEIMDAL at the ESS, Lund and iMATERIA at J-PARC can be relevant examples of instruments designed for studying with SANS advanced functional materials, including fibers $[19,20]$.

In this paper, we will present WAXS data collected on three cases of technological interest (type I collagen, silk fibroin extracted from Bombyx mori and cellulose), aimed at their molecular study.

Type I collagen. Type I collagen is the main fibril-forming protein of the connectives, representing approximately $70 \%$ of the total collagen in mammals [21,22]. It is distributed within a wide range of tissues, such as bone and skin, and confers biomechanical elasticity and support to tissues. Thanks to its biocompatibility and non-toxic degradability, it is widely used in several biomedical fields [23] and it is considered one of the gold standard biomaterials for scaffolds fabrication in regenerative medicine. Type I collagen is a complex hierarchically organized structure characterized by three $\alpha$-helices, two $\alpha 1$ (I) chains, encoded by collagen type I alpha 1 chain gene (COL1A1), and one $\alpha 2$ (I) chain, encoded by COL1A2 gene [24], intertwined in a typical heterotrimeric molecule of $300 \mathrm{~nm}$ in length and $1.5 \mathrm{~nm}$ in diameter [25]. In the triple helix, three sections are clearly distinguishable: a central helical part with the repetition of the characteristic triplet Gly-X-Y, and two non-helical regions at $C$ and N-termini (telopeptides). The tightly packed conformation of the triple helix is guaranteed by the glycine amino acid retained in every third position of the triplet, while Pro and Hyp, often observed in $X$ and $Y$ positions, are placed on the helical surface, available for steric interactions with other molecules. Furthermore, the telopeptides placed at the end of the helical region comprise the sites for intra- and intermolecular crosslinks (trivalent pyridinoline or pyrrole crosslinks at the $\mathrm{N}$-terminus and divalent intermolecular crosslinks at the C-termimus) that are crucial for collagen supramolecular organization and strength. Collagens triple helices are arranged in fibril bundles due to electrostatic and hydrophobic interactions, with a variable diameter $(10-500 \mathrm{~nm})$, characterized by a molecular distance of about $1.6 \mathrm{~nm}$, that varies in relation to the tissue and the hydration state. Indeed, the molecular structure is stabilized by water-mediated or direct hydrogen bonds, between the NH group on the glycine backbone and the $\mathrm{CO}$ group on the backbone of the amino acid in $\mathrm{X}$ position of the triplet Gly-X-Y, placed on the closed chain. The creation of a cylinder of hydration permits the stabilization of the crystal lattice of collagen. Fibrils are arranged in longitudinally staggered arrays, in which each of them is staggered about one-quarter of its length with respect to the neighbor along the axis of the fibril. This disposition gives rise to a gap $(0.54 \bullet \mathrm{d})$ - overlap $(0.46 \bullet \mathrm{d})$ nanoscale structure (regions of high and low electron density) showing a d-periodic banding of 64-67 nm axial periodicity [16]. Fibrils are also packed into fibers with a final diameter of about $100 \mathrm{~nm}$, depending on hydration state and tissue. Indeed, as type I collagen can be extracted from different sources, it partially retains tissue-related features, depending on the particular extraction process. It is mainly isolated from fish scales and skin [26], bovine dermis and equine tendons [27] with a variable yield. The main issue related to the protein isolation from the tissues is obtaining a soluble fraction of protein, which is related to the age of the animal. Thus, younger tissues are preferred, as they allow to one extract the higher amount of soluble protein, about $2 \%$. The extraction can be performed with chemical or enzymatic hydrolysis. Among all the extraction protocols, the chemical method ensures the preservation of the molecular 
conformation and the partial retention of the nanofibrillary structure. On the contrary, the enzymatic protocol, consisting of the selective cleavage of the telopeptides at both the $\mathrm{N}$ - and C-termini, permits researchers to obtain smaller molecules, named atelocollagen, with a low retention of fibrillary structure, as the telopeptides containing crosslinking sites are cleaved. However, molecules are still bonded with few intermolecular crosslinks along the triple helical length. The achieved atelocollagen induces a lower immune response, as the telopeptides also comprise the antigenic P-determinant [28]. Thus, the retention of few crosslinks between molecules is related to the type of tissue and extraction methods. In particular, the stiffer the extraction tissue, the more the fibrillary structure is retained.

Silk fibroin (Bombyx mori). Silk fibroin (SF) is a natural material derived from Bombyx mori cocoons. This biopolymer, in its native or regenerated forms, is largely used and studied for biomaterial applications thanks to its excellent properties like mechanical strength, elasticity, biocompatibility and biodegradability, versatile processability, ease of functionalization and thermal stability. In addition, silk fibroin possesses good optical properties like transparency in the visible range together with a remarkable surface smoothness, making it a suitable material for the processing of photonic devices for biomedical applications. The main fields of applications are medical/pharmaceutical, tissue engineering, drug delivery, optics, sensing and diagnostics. The morphologies, together with the peculiar macroscopic properties of silk fibroin, are strictly related to its molecular structure, which has been extensively studied in several papers since 1913 by Nishikawa and Ono [29] by X-ray diffraction, investigating the hierarchical structure of silk fibers. The $\mathrm{X}$-ray structural analysis of the silk fibroin allows one to determine the structural features like unit cell dimension, nanocrystallite size and orientation, and degree of crystallinity. In 1955, the crystal structure of silk fibroin was proposed by Marsh, Corey and Pauling [30] as a regular arrangement of antiparallel $\beta$-sheets.

From the molecular structural point of view, the silk fibroin is a protein composed by four aminoacids: glycine $(43.7 \%)$, alanine $(28.8 \%)$, serine $(11.9 \%)$ and tyrosine $(5 \%)$ arranged in heavy-chain $(\mathrm{H}), 390 \mathrm{kDa}$, and light-chain $(\mathrm{L}), 26 \mathrm{kDa}$, linked together by a disulfide bond [31] at the C-terminus of $\mathrm{H}$-chain, forming an $\mathrm{H}-\mathrm{L}$ complex. In particular, $\mathrm{H}$-chains form small discrete $\beta$-sheet crystallites embedded in an amorphous matrix, which constitutes the main structural component conferring the exceptional mechanical properties to this biopolymer (hydrophobic region).

The size of $\beta$-sheet crystallites was estimated to be $2.6 \mathrm{~nm}$ in the intermolecular hydrogen bond direction, $32.0 \mathrm{~nm}$ in the sheet stacking direction and $11.5 \mathrm{~nm}$ in the chain direction, respectively. The hydrophobic regions of $S F$ interact with each other, giving rise to supramolecular nanocrystallites which adopt an orthorhombic structure with unit cell dimensions $\mathrm{a}=9.20 \AA, \mathrm{b}=9.40 \AA$ and $\mathrm{c}=6.97 \AA$ [32-34]. The hydrophilic disordered regions allow for the aqueous solubility of the material.

The degree of crystallinity can be estimated by analyzing the crystalline and amorphous components present in the WAXS profiles. Different methods have been proposed and applied for such analysis with results in good agreement for silk fibroin from Bombyx mori that show a crystallinity of $45.6 \%$, higher than all other silks, indicating a higher abundance of nanocrystalline component in Bombyx mori silk [35].

The study of the structure-function relationship is one of the main research topics in silk-based polymers since it determines fields and limits of its application. The interesting properties of silk fibroin are thought to originate from the particular molecular structures of the material. In particular the Young's modulus of silk is enhanced with increasing $\beta$-sheet content and crystallinity. Indeed, Bombyx mori silk fibroin shows the highest tensile strength and Young's modulus compared to other types of silk.

Cellulose. Cellulose is a glucose polymer, insoluble in water, with a hierarchically ordered structure composed by a crystalline and an amorphous component. There are different crystalline polymorphs of cellulose [36], but the most known are cellulose I (with the two allomorphs, triclinic I $\alpha$ and monoclinic I $\beta[37,38]$, which corresponds to native cellulose) and cellulose II, obtained by chemical regeneration or mercerization of natural cellulose [38]. The term fiber usually refers to a bundle of cells adhering to each other [39]. The cellulose I $\beta$ is thermodynamically more stable. [40]. In cellulose, glucose 
molecules are linked together by $\beta(1-4)$ linkages, such that each glucose molecule is rotated $180^{\circ}$ with respect to the other. Two $180^{\circ}$-linked glucose molecules form a cellobiose unit, which is the repeat unit of cellulose. Cellobiose units are linked together, forming cellulose chains, whose length or degree of polymerization - number of glucose monomers-is variable, up to about 10,000 units in the secondary walls of the fibers [41]. Cellulose chains are linked together to form microfibrils. Various hypotheses have been proposed regarding the cross-sectional arrangement of microfibrils [39]. In particular, a microfibril of 18-24 chains should be $2-3 \mathrm{~nm}$ wide, leading to main X-Ray scattering signals in the SAXS regime. Microfibrils aggregate together, forming macrofibrils [42], up to a maximum cross-sectional size of about $50 \mathrm{~nm}$, although further studies are needed to understand the exact structure of microfibrils and macrofibrils. Indeed, the model of cellulose microfibrils, linked together within a hemicellulose and lignin matrix, is an approximate description of the textile fibers. As an example, we report the composition of some natural textiles: cotton fiber contains $94-96 \%$ of pure cellulose and $4-6 \%$ of other components; flax/linen fibers contain $92 \%$ of pure cellulose, $2 \%$ of hemicellulose, $2 \%$ of lignin and $4 \%$ of other components; hemp fibers contain $78 \%$ of pure cellulose, $10 \%$ of hemicellulose, $7 \%$ of lignin and $5 \%$ of other components. Wood contains $50 \%$ of cellulose.

In this approximate model, textile fibers' cellulose can be divided into "well-ordered" crystal-surface and "poorly-ordered" crystal-interior cellulose [43], showing also the absence of any order (amorphous state). These partially ordered structures lead to scattering signals in the WAXS regime, from which information about the degree of crystallinity of the fibers can be extracted. Amorphous cellulose is the result of mechanical/chemical damage of crystalline cellulose, rather than being a native state [39]. Thus, the WAXS patterns contain specific features of the fibrous samples and of its state of integrity and conservation.

\section{Materials and Methods}

Type I collagen. The type I collagen here analyzed was derived by commercially available raw flakes extracted from equine tendons through a chemical protocol. The protein was provided by Typeone Srl (Lecce, Italy) [44,45].

Silk fibroin from Bombyx mori. The degummed silk fibroin was obtained from Bombyx mori cocoons purchased from TaJima Shoji (Japan). The sericin was removed from the silk fibroin cocoons by cutting them into fourths, then the silk fibroin was shredded and boiled for $30 \mathrm{~min}$ in aqueous solution of $\mathrm{Na}_{2} \mathrm{CO}_{3} 0.02 \mathrm{M}$ and then rinsed with bi-distilled water to remove residual sericin and excess of salt. The fiber was then dried for $24 \mathrm{~h}$ at ambient conditions.

Cellulose. The cellulose sample was a linen thread with a length of about $1 \mathrm{~cm}$ and a thickness of around $0.2-0.4 \mathrm{~mm}$. This sample was taken form a group of fabrics analyzed by WAXS with the aim to offer an alternative method to radiocarbon dating, entirely based on X-ray data [46].

The fiber diffraction patterns were collected with a dedicated set-up (X-ray MicroImaging Laboratory, XMI-L@b) shown in Figure 2a. The micro-source, a Fr-E+ SuperBright rotating copper anode microsource ( $45 \mathrm{kV} / 55 \mathrm{~mA} ; \mathrm{Cu} \mathrm{K} \alpha, \lambda=0.154 \mathrm{~nm}, 2475 \mathrm{~W})$, displayed in Figure 2b,c, was coupled through a multilayer focusing optics (Confocal Max-Flux; CMF 15-105) to a three-pinhole camera (Rigaku SMAX3000) for small- and wide-angle X-ray scattering (SAXS/WAXS) data collection. The samples were placed in the sample chamber (Figure $2 \mathrm{~d}$ ) and measured in vacuum (0.1-1 mbar). A goniometer with a $125 \times 125 \mathrm{~mm}^{2}$ stage can be inserted into the chamber to collect grazing incidence small- and wide-angle scattering data (GI-SAXS/GI-WAXS). For WAXS/GIWAXS data collection, an image-plate (IP) detector $\left(250 \times 160 \mathrm{~mm}^{2}\right.$, with 50 or $100 \mu \mathrm{m}$ effective pixel size depending on binning, and off-line RAXIA reader) was inserted at $\sim 28 \mathrm{~mm}$ from the sample, using a load-lock system (Figure 2e) which allowed the mounting and demounting of the detector without breaking the vacuum. Alternatively, the same detector could be mounted into the sample chamber at other distances up to $250 \mathrm{~mm}$ from the sample. The set-up was equipped with a second detector, a Triton 20 gas-filled proportional counter $(1024 \times 1024$ array, $195 \mathrm{~mm}$ pixel size) for SAXS/GISAXS acquisition. 
The two detectors could also be used simultaneously thanks to a $6 \mathrm{~mm}$ hole in the center of the WAXS detector $[47,48]$.

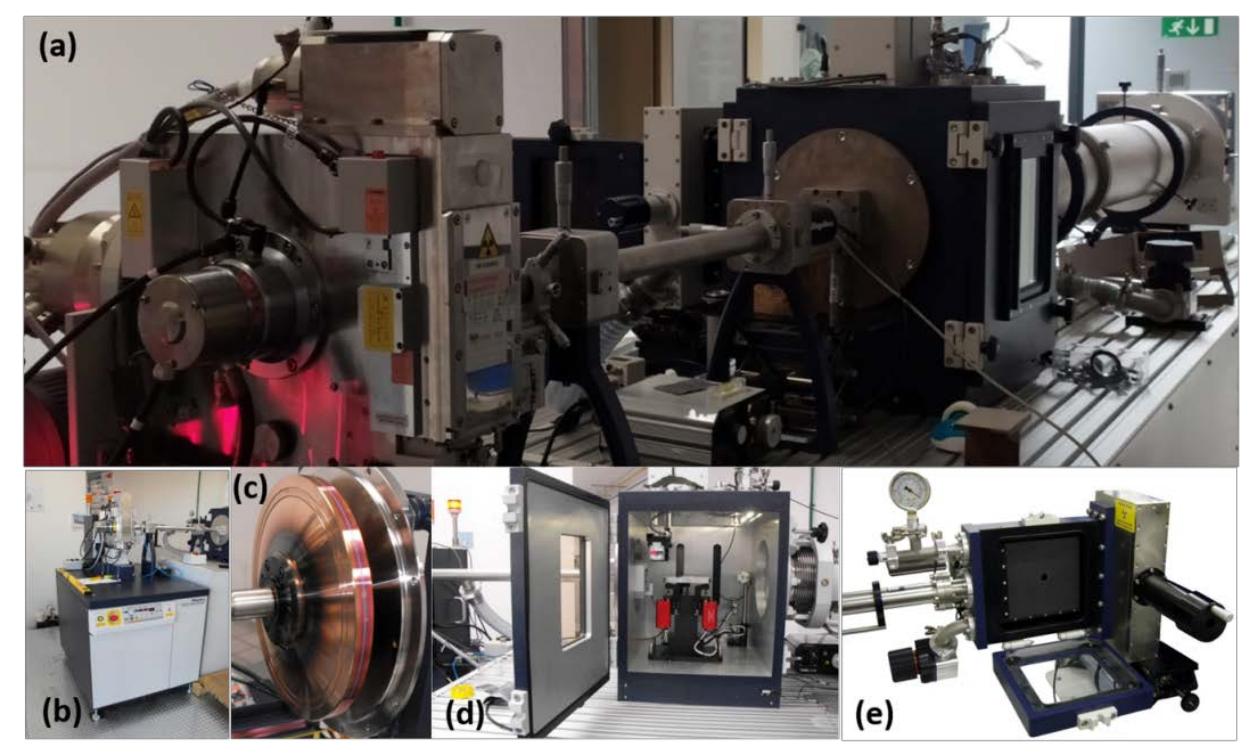

Figure 2. (a) X-ray MicroImaging Laboratory (XMI-L@b) set-up; in details: (b) Fr-E+ SuperBright microsource; (c) Cu rotating anode; (d) the sample chamber; (e) load-lock system for mounting/demounting the image plate detector for wide-angle X-ray scattering (WAXS)/grazing incidence wide-angle scattering (GIWAXS) data collection.

\section{Results and Discussion}

\subsection{Type I Collagen}

Two models for type I collagen have been reported in the literature: the 10/3 model, described by Rich and Crick [49], characterized by the pitch length of $86 \AA$ and 3.33 amino acidic residues per turn that confer the axial repetition of $28.8 \AA$ and the $7 / 2$ model, described by Okuyama et al. [50], with the pitch length of $60 \AA$ and 3.5 amino acidic residues per turn, conferring the axial repetition of $20.0 \AA$ to the molecule. Figure 3 shows the WAXS data collected on type 1 collagen (Figure 3a) and the 2d3h molecular model (Figure 3b) [51] reported in the protein database, which keeps a triple-helical structure very close to the ideal 7/2-helical model. The 2D WAXS pattern displays the typical cross $\beta$ fiber diffraction features with the intensity distributed along two main orthogonal directions, the meridional one along the fiber axis and the equatorial one perpendicular to it. The pattern is centered, calibrated and integrated along the meridional and equatorial directions, and the corresponding 1D WAXS profiles are displayed in the same figure (yellow curves). 


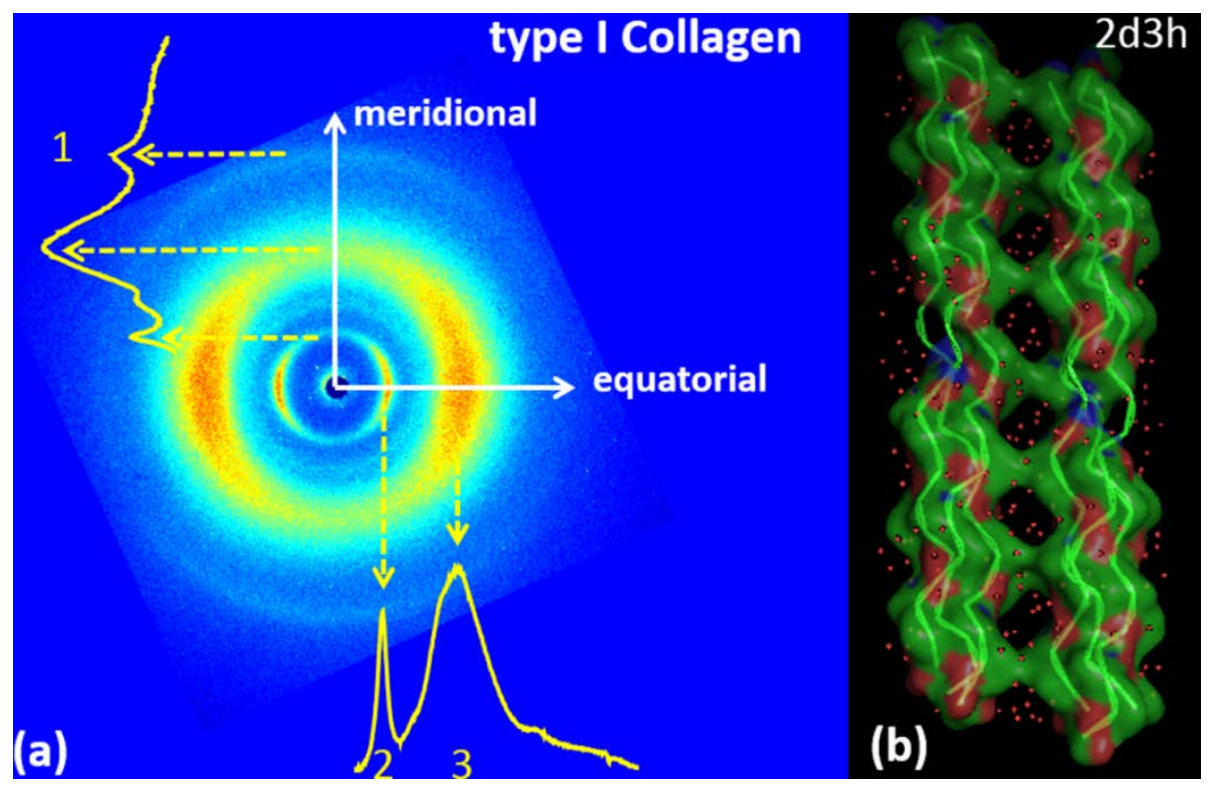

Figure 3. (a) Two-dimensional WAXS fiber diffraction pattern of type I collagen with white arrows along the fiber axis (meridional) and perpendicular to it (equatorial). The corresponding 1D WAXS integrated profiles show the main peaks of the protein, the meridional peak (marked as 1) and the equatorial peaks (marked as 2 and 3), respectively; (b) 2d3h molecular model of type I collagen.

As shown in Table 1, as well as in our recent WAXS studies performed on type I collagen [44,45], we found a meridional diffraction peak at $\mathrm{q}_{1}=2.22 \pm 0.075 \AA^{-1}$ (marked as 1 in Figure 3a), corresponding to the distance between amino acidic residues along the c-axis of the helix $(\mathrm{d}=2.8 \pm 0.1 \AA$, helical axial periodicity). A further diffraction signal was found orthogonally to it: the equatorial peak at $\mathrm{q}_{2}=0.6 \pm 0.05 \AA^{-1}$ (marked as 2 in Figure 3a), corresponding to the lateral packing $(\mathrm{d}=10.65 \pm 1 \AA$ ) of triple helices which varies with the hydration state of the material. Along the equatorial direction was also observed a broad amorphous-like peak at $q_{3}=1.39 \pm 0.25 \AA^{-1}$ (marked as 3 in Figure 3a), corresponding to $\mathrm{d}=4.55 \pm 0.85 \AA$ and describing the distance between collagen skeletons. The stability of the triple helices is increased by the formation of direct hydrogen bonds between the $\mathrm{NH}$ group of glycine and the $\mathrm{CO}$ group of the amino acid in the $\mathrm{X}$ position on the neighboring chain, and it is also enhanced by the formation of a hydration cylinder around the molecular configuration. Thus, the hydration state of the material is important for the lateral packing of collagen molecules in the crystal lattice arrangement and may affect the equatorial peak position [52]. Indeed, it was demonstrated that collagen concentrations larger than $30-40 \mathrm{mg} / \mathrm{mL}$ allow one to arrange triple helices in liquid crystal phases with two oriented preferred geometries: nematic (not arranged in layers) and cholesteric (arranged in layers) [53]. By measuring the exact positions of meridional and equatorial peaks, relevant information on the stress state of the fiber can be extracted.

\subsection{Silk Fibroin from Bombyx Mori}

Figure 4 shows the WAXS data (Figure 4a) collected on fibroin-Bombyx mori and the 2slk molecular model [54] reported in the Protein Data Bank (PDB) protein database for this fiber (Figure 4b). The 2D WAXS pattern displays the typical cross $\beta$ fiber diffraction features with the intensity distributed along two main orthogonal directions, the meridional along the fiber axis and the equatorial perpendicular to it. The pattern is centered, calibrated and integrated along the meridional and equatorial directions, and the corresponding 1D WAXS profiles are displayed in the same figure (yellow curves). The positions of the main peaks are reported in Table 1. 


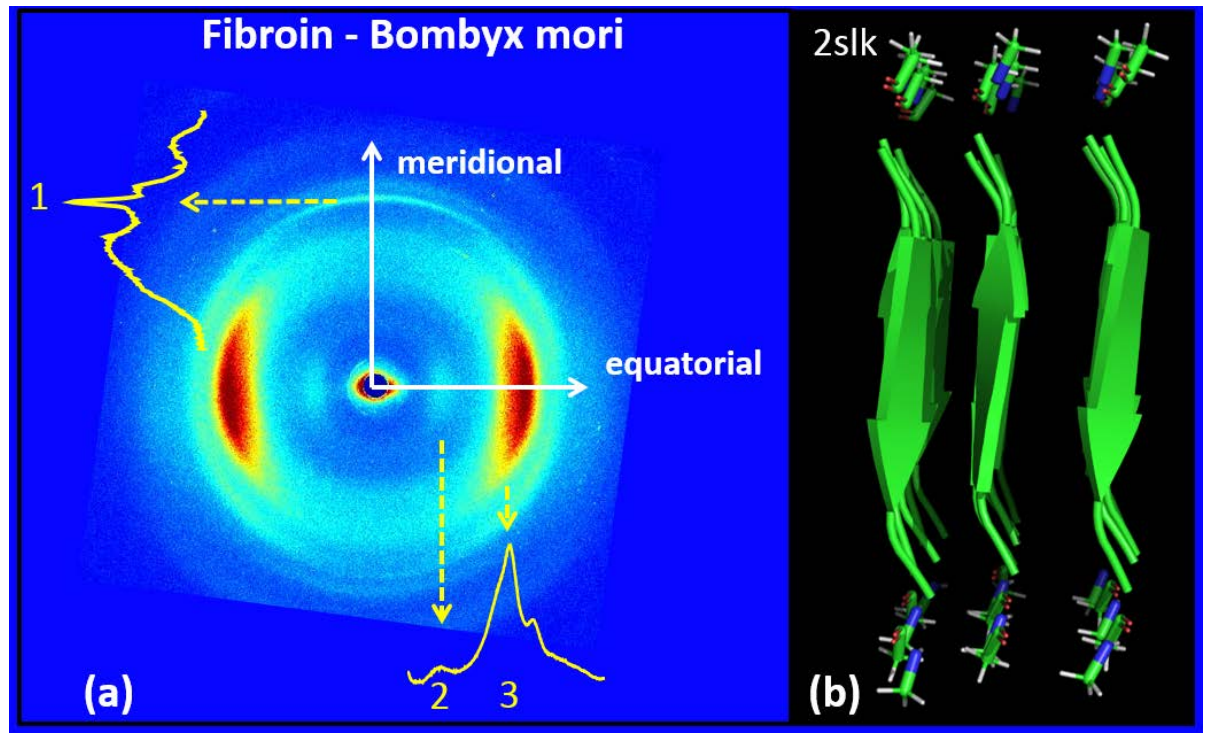

Figure 4. (a) Two-dimensional WAXS fiber diffraction pattern collected on fibroin-Bombyx mori with white arrows along the fiber axis (meridional) and that one perpendicular to it (equatorial). The corresponding 1D WAXS integrated profiles show the main peaks of the protein, the meridional peak (marked as 1) and the equatorial peaks (marked as 2 and 3), respectively; (b) 2slk molecular model of fibroin-Bombyx mori.

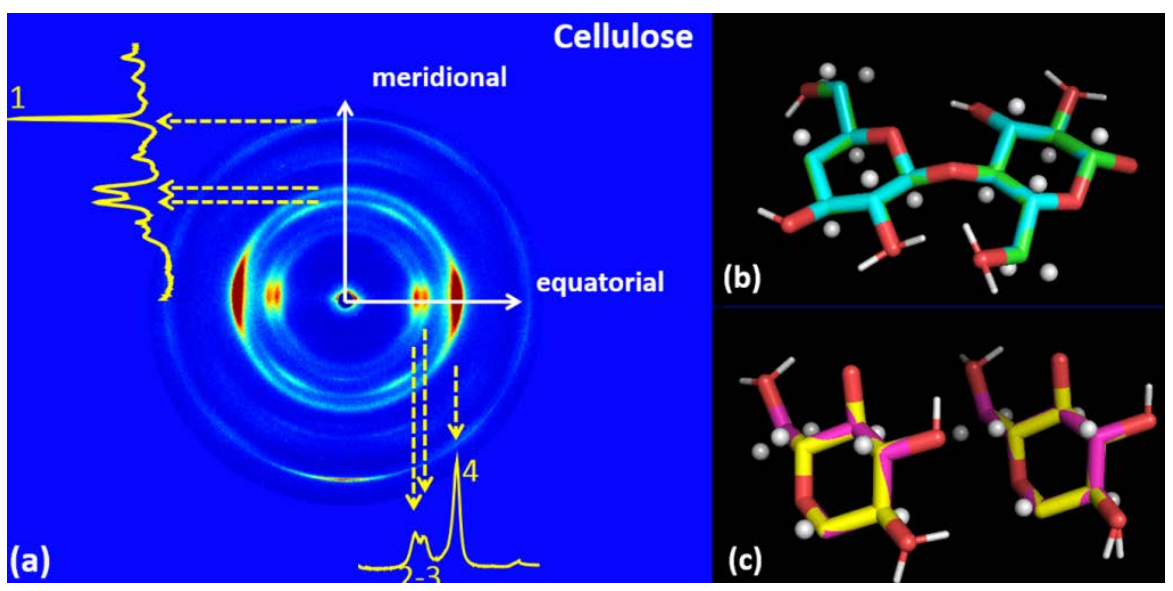

Figure 5. (a) Two-dimensional WAXS fiber diffraction pattern collected on cellulose [46], with white arrows along the fiber axis (meridional) and perpendicular to it (equatorial). The corresponding 1D WAXS integrated profiles show the main peaks of the protein, i.e., the meridional peak (marked as 1) and the equatorial peaks (marked as 2, 3 and 4), respectively; (b) I $\alpha$ model; (c) I $\beta$ model.

The meridional peak at $\mathrm{q}_{1}=1.78 \pm 0.03 \AA^{-1}$ (marked as 1 in Figure $4 \mathrm{a}$ ), corresponding to an interplanar distance $\mathrm{d}_{1}=3.53 \pm 0.06 \AA$, was correctly indexed as the (002) reflection of the Silk I [55]. Indeed, as reported in the literature [56], this indicates that the $\beta$ strands along the fiber axis yield the $7.0 \AA$ axial repeat and contain two peptide units. The equatorial peaks indicate the distance along the intersheet and interchain direction. The equatorial peak at $\mathrm{q}_{2}=0.66 \pm 0.075 \AA^{-1}$ (marked as 2 in Figure $4 a$ ) corresponding to $d_{2}=9.52 \pm 1 \AA$ was indexed as the (010) reflection, whereas the peak at $\mathrm{q}_{3}=1.44 \pm 0.08 \AA^{-1}$ (marked as 3 in Figure $4 \mathrm{a}$ ), corresponding to $\mathrm{d}_{3}=4.36 \pm 0.25 \AA$, was indexed as an overlap of the (020) and (210) reflections. The unit cell determined from the d-spacing values reflection (020), (210) and (002) shows a good agreement with the literature [55]. 
Table 1. Positions and relative interplanar distances extracted from the 1D-WAXS data reported in Figure 3 (TYPE 1 COLLAGEN), Figure 4 (FIBROIN-BOMBYX MORI) and Figure 5 (CELLULOSE).

\begin{tabular}{cc}
\hline & TYPE 1 COLLAGEN \\
\hline $\mathrm{q}_{1}=2.22 \pm 0.075 \AA^{-1} ; \mathrm{d}_{1}=2.83 \pm 0.1 \AA$ & $\mathrm{q}_{2}=0.59 \pm 0.05 \AA^{-1} ; \mathrm{d}_{2}=10.65 \pm 1 \AA$ \\
\hline & $\mathrm{q}_{3}=1.39 \pm 0.25 \AA^{-1} ; \mathrm{d}_{3}=4.52 \pm 0.85 \AA$ \\
\hline $\mathrm{q}_{1}=1.78 \pm 0.03 \AA^{-1} ; \mathrm{d}_{1}=3.53 \pm 0.06 \AA$ & $\mathrm{q}_{2}=0.66 \pm 0.075 \AA^{-1} ; \mathrm{d}_{2}=9.52 \pm 1 \AA$ \\
\hline & $\mathrm{q}_{3}=1.44 \pm 0.08 \AA^{-1} ; \mathrm{d}_{3}=4.36 \pm 0.25 \AA$ \\
\hline $\mathrm{q}_{1}=2.43 \pm 0.015 \AA^{-1} ; \mathrm{d}_{1}=2.58 \pm 0.01 \AA$ & $\mathrm{q}_{2}=1.05 \pm 0.04 \AA^{-1} ; \mathrm{d}_{2}=6.00 \pm 0.25 \AA$ \\
\hline & $\mathrm{q}_{3}=1.18 \pm 0.025 \AA^{-1} ; \mathrm{d}_{3}=5.32 \pm 0.15 \AA$ \\
\hline
\end{tabular}

\subsection{Cellulose}

Figure 5 shows the WAXS data (Figure 5a) collected on linen cellulose. Unlike the previous two cases, we could not find a single molecular model to describe the hierarchical fiber organization. We report here the two crystal structures of cellulose $\mathrm{I} \alpha$ (Figure $5 b$ ) and I $\beta$ (Figure $5 c$ ) which better explain our WAXS data $[35,36]$. As with the previous examples, the 2D WAXS pattern displays the typical fiber diffraction features with the intensity distributed along two main orthogonal directions, the meridional one along the fiber axis and the equatorial one perpendicular to it. The pattern is centered, calibrated and integrated along the meridional and equatorial directions, and the corresponding 1D WAXS profiles are displayed in the same figure (yellow curves). The positions of the main peaks are reported in Table 1.

The equatorial peaks at $\mathrm{q}_{2}=1.05 \pm 0.04 \AA^{-1}$ and $\mathrm{q}_{3}=1.39 \pm 0.25 \AA^{-1}$, marked as 2 and 3 , respectively, in Figure 5a can be indexed as the (010) and (001) reflections in the case of the triclinic cellulose I $\alpha$ phase or alternatively as the (1-10) and (110) reflections in the case of the monoclinic cellulose $\mathrm{I} \beta$ phase; the meridional peak at $\mathrm{q}_{1}=2.43 \pm 0.015 \AA^{-1}$, marked as 1 in Figure $5 \mathrm{a}$, can be indexed as the (021) reflection in the case of the triclinic cellulose I $\alpha$ phase or alternatively as the (004) reflection in the case of monoclinic cellulose I $\beta$ phase $[57,58]$.

\section{Conclusions and Perspectives}

Engineered polymer fibers are extensively used in biomedical applications thanks to the high similarity with the extracellular matrix, the outstanding mechanical properties and the high versatility. In these fields, both natural (collagen, silk, cellulose, elastin, chitosan, alginate) and synthetic (polylactic acid-PLA, poly-lactic-co-glycolic acid-PLGA, polycaprolactone-PCL) fibrous polymers are used to fabricate fiber networks for cells and to improve their mechanical response.

The protocols of extraction and/or fabrication of the devices based on natural or engineered fibers are numerous. For each of them, it is necessary to correlate specific functions to the structural organization of the fiber. Fiber polymers have often a complex hierarchical structure with well-defined organization on multiple length scales from sub-molecular to supramolecular scale. To gain deeper insight into the assembly process, $\mathrm{X}$-ray scattering techniques allow inspection of the material from atomic/molecular (WAXS) organization to nanoscale (SAXS), i.e., the whole hierarchical architecture.

In this paper, we report the WAXS investigation performed with a table-top high brilliance $\mathrm{X}$-ray micro-source on three different types of natural fibers (collagen, silk fibroin and cellulose) 
aiming to show the power of fiber wide-angle X-ray scattering for the molecular characterization of natural polymers. For many biological/natural systems, the fibrous state is functionally crucial to a specific function.

Besides the cases of natural polymers here reported, novel studies have been reported on other materials, for example aromatic peptide-based materials that exhibit spontaneous phenomena of supramolecular organization into ordered nanostructures [59-61]. Here, the knowledge of their inner molecular organization was correlated to the design of novel molecules with enhanced and tunable properties for drug delivery or contrast agents.

Author Contributions: T.S., A.T., D.A., R.L. and C.G. realized the WAXS experiments at the XMI-L@b, L.D.C., M.L., F.S. and D.S. wrote new algorithms for WAXS data analysis and implemented them into the SUNBIM software which was used to analyze the experimental data. A.M. indexed the WAXS patterns. T.S., A.T, L.D.C. and C.G. discussed the results and wrote the paper in close collaboration with all the authors. All authors have read and agree to the published version of the manuscript.

Funding: This work was partially supported by SAPIENT project "A system approach for identifying connective tissue degeneration in diabetic analogues (CUP: B54I19002520001)"; by the ISEPA project "Improving Sustainability, Efficiency and Profitability of Large Scale Aquaponics" (CUP: B37H17004760007) funded by Regione Puglia in the framework of InnoNetwork. Sostegno alle attività di RES per lo sviluppo di nuove tecnologie sostenibili, di nuovi prodotti e servizi and by PON MIUR project "Energy for TARANTO" (Proposal Code ARS01_00637).

Acknowledgments: Luca Salvatore (TypeOne Srl, Lecce), Gianluca Maria Farinola (University of Bari) and Giulio Fanti (University of Padova) are acknowledged for providing samples for WAXS tests. Giovanni Filograsso, Brunella Aresta, Caterina Chiarella and Lucrezia Cassano are acknowledged for their contribution in the projects administration.

Conflicts of Interest: The authors declare no conflict of interest.

\section{References}

1. Nguyen, L.T.H.; Chen, S.; Elumalai, N.K.; Prabhakaran, M.P.; Zong, Y.; Vijila, C.; Allakhverdiev, S.I.; Ramakrishna, S. Biological, Chemical, and Electronic Applications of Nanofibers, Macromol. Mater. Eng. 2013, 298, 822-867.

2. Ko, F.K.; Wan, Y. Introduction to Nanofiber Materials; Cambridge University Press: Cambridge, UK, 2014.

3. Nemati, S.; Kim, S.; Shin, Y.M.; Shin, H. Current progress in application of polymeric nanofibers to tissue engineering. Nano Converg. 2019, 6, 36. [CrossRef] [PubMed]

4. Obaid, M.; Barakat, N.A.; Fadali, O.A.; Al-Meer, S.; Elsaid, K.; Khalil, K.A. Stable and effective super-hydrophilic polysulfone nanofiber mats for oil/water separation. Polymer 2015, 72, 125-133. [CrossRef]

5. Cheng, L.; Ma, S.Y.; Wang, T.T.; Li, X.B.; Luo, J.; Li, W.Q.; Mao, Y.Z.D.; GZ, J. Synthesis and characterization of $\mathrm{SnO}_{2}$ hollow nanofibers by electrospinning for ethanol sensing properties. Mater. Lett. 2014, 131, $23-26$. [CrossRef]

6. Chen, F.L.; Huang, H.Z.; Liang, W.H.; Guan, F.Q.; Yu, H.S. Bacterial-cellulose-derived carbon nanofiber $\mathrm{MnO}_{2}$ and nitrogen-doped carbon nanofiber electrode materials: An asymmetric supercapacitor with high energy and power density. Adv. Mater. 2013, 25, 4746-4752. [CrossRef]

7. Altamura, D.; Pastore, S.G.; Raucci, M.G.; Siliqi, D.; De Pascalis, F.; Nacucchi, M.; Ambrosio, L.; Giannini, C. Scanning small- and wide-angle X-ray scattering microscopy selectively probes ha content in gelatin/hydroxyapatite scaffolds for osteochondral defect repair. ACS Appl. Mater. Interfaces 2016, 8, 8728-8736. [CrossRef]

8. O'brien, F.J. Biomaterials \& scaffolds for tissue engineering. Mater. Today 2011, 14, 88.

9. Schneider, A.; Wang, X.Y.; Kaplan, D.L.; Garlick, J.A.; Egles, C. Biofunctionalized electrospun silk mats as a topical bioactive dressing for accelerated wound healing. Acta Biomater. 2009, 5, 2570-2578. [CrossRef]

10. Wan, Y.Q.; He, J.H.; Yu, J.Y. Carbon nanotube-reingorced polyacrylonitrile nanofibers by vibration-electrospinning. Polym. Int. 2007, 56, 1367-1370. [CrossRef]

11. Ranganath, A.S.; Ganesh, V.A.; Sopiha, K.; Sahay, R.; Baji, A. Investigation of wettability and moisture sorption property of electrospun poly (N-isopropylacrylamide) nanofibers. MRS Adv. 2016, 1, 1959-1964. [CrossRef]

12. Ambrosi, A.; Sasaki, T.; Pumera, M. Platelet graphite nanofibers for electrochemical sensing and biosensing: The influence of graphene sheet orientation. Chem. Asian. J. 2010, 5, 266-271. [CrossRef] [PubMed] 
13. Terzi, A.; Gallo, N.; Bettini, S.; Sibillano, T.; Altamura, D.; Madaghiele, M.; De Caro, L.; Valli, L.; Salvatore, L.; Sannino, A.; et al. Sub and supramolecular X-ray characterization of engineered tissues from equine tendons, bovine dermis and fish skin type-I collagen. Macromol. Biosci. 2020. [CrossRef] [PubMed]

14. Sibillano, T.; De Caro, L.; Scattarella, F.; Scarcelli, G.; Siliqi, D.; Altamura, D.; Liebi, M.; Ladisa, M.; Bunk, O.; Giannini, C. Interfibrillar packing of bovine cornea by table-top and synchrotron scanning SAXS microscopy. J. Appl. Cryst. 2016, 49, 1231-1239. [CrossRef]

15. Giannini, C.; DSiliqi, D.; Ladisa, M.; Altamura, D.; Diaz, A.; Beraudi, A.; Sibillano, T.; De Caro, L.; Stea, S.; Baruffaldi, F.; et al. Scanning SAXS-WAXS microscopy on osteoarthritis-affected bone-An age-related study. J. Appl. Cryst. 2014, 47, 110-117. [CrossRef]

16. Giannini, C.; Terzi, A.; Fusaro, L.; Sibillano, T.; Diaz, A.; Ramella, M.; Lutz-Bueno, V.; Boccafoschi, F.; Bunk, O. Scanning X-ray microdiffraction of decellularized pericardium tissue at increasing glucose concentration. J. Biophotonics 2019, 12, e201900106. [CrossRef]

17. Giannini, C.; Ladisa, M.; Lutz, V.-B.; Terzi, A.; Ramella, M.; Fusaro, L.; Altamura, D.; Siliqi, D.; Sibillano, T.; Diaz, A.; et al. X-ray scanning microscopies of microcalcifications in abdominal aortic and popliteal artery aneurysms. IUCrJ 2019, 6, 267-276. [CrossRef]

18. Diaferia, C.; Mercurio, F.A.; Giannini, C.; Sibillano, T.; Morelli, G.; Leone, M.; Accardo, A. Self-assembly of PEGylated tetra-phenylalanine derivatives: Structural insights from solution and solid state studies. Sci. Rep. 2019, 6, 26638. [CrossRef]

19. Viell, J.; Inouye, H.; Szekely, N.K.; Frielinghaus, H.; Marks, C.; Wang, Y.; Anders, N.; Spiess, A.C.; Makowski, L. Multi-scale processes of beech wood disintegration and pretreatment with 1-ethyl-3-methylimidazolium acetate/water mixtures. Biotechnol. Biofuels 2016, 9, 7. [CrossRef]

20. Liao, X.; Dulle, M.; Martins de Souza e Silva, J.; Wehrspohn, R.B.; Agarwal, S.; Förster, S.; Hou, H.; Smith, P.; Greiner, A. High strength in combination with high toughness in robust and sustainable polymeric materials. Science 2019, 366, 1376-1379. [CrossRef]

21. Adachi, E.; Hopkinson, I.; Hayashi, T. Basement-Membrane Stromal Relationships: Interactions between Collagen Fibrils and the Lamina Densa. Int. Rev. Cytol. 1997, 173, 73-156.

22. Sorushanova, A.; Delgado, L.M.; Wu, Z.; Shologu, N.; Kshirsagar, A.; Raghunath, R.; Mullen, A.M.; Bayon, Y.; Pandit, A.; Raghunath, M.; et al. The Collagen Suprafamily: From Biosynthesis to Advanced Biomaterial Development. Adv. Mat. 2019, 31, 1801651. [CrossRef] [PubMed]

23. Parenteau-Bareil, R.; Gauvin, R.; Berthod, F. Collagen-Based Biomaterials for Tissue Engineering. Appl. Mat. 2010, 3, 1863-1887.

24. Persikov, A.V.; Pillitteri, R.J.; Amin, P.; Schwarze, U.; Byers, P.H.; Brodsky, B. Stability related bias in residues replacing glycines within the collagen triple helix (Gly-Xaa-Yaa) in inherited connective tissue disorders. Human Mutat. 2004, 24, 330-337. [CrossRef] [PubMed]

25. Ramachandran, G.N.; Kartha, G. Structure of Collagen. Nature 1955, 176, 593-595. [CrossRef] [PubMed]

26. Cen, L.; Liu, W.; Cui, L.; Zhang, W.; Cao, Y. Collagen tissue engineering: Development of novel biomaterials and applications. Pediatr Res. 2008, 63, 492-496. [CrossRef]

27. Nimni, M.E.; Cheung, D.; Strates, B.; Kodama, M.; Sheikh, K. Chemically modified collagen: A natural biomaterial for tissue replacement. Biomed. Mat. Res. 1987, 21, 741-771. [CrossRef] [PubMed]

28. Knapp, T.R.; Luck, E.; Daniels, J.R. Behavior of solubilized collagen as a bioimplant. J. Surg. Res. 1977, 23, 96-105. [CrossRef]

29. Nishikawa, S.; Ono, S. Transmission of X-rays through fibrous, lamellar and granular substances. Proc. Tokyo Math Phys. Soc. 1913, 7, 131.

30. Marsh, R.E.; Corey, R.B.; Pauling, L. An investigation of the structure of silk Fibroin. Biochim. Biophys. Acta 1955, 16, 1. [CrossRef]

31. Shimura, K. Chemical composition and biosynthesis of silk proteins. Experimentia 1983, 39, 455. [CrossRef]

32. Takashi, Y.; Gehoh, M.; Yuzuriha, K. Structure refinement and diffuse streak scattering of silk (Bombyx mori). Int. J. Biol. Macromol. 1999, 24, 127-138. [CrossRef]

33. Asakura, T.; Ohata, T.; Kametani, S.; Okushita, K.; Yazawa, K.; Nishiyama, Y.; Nishimura, K.; Aoki, A.; Suzuki, F.; Kaji, H.; et al. Intermolecular packing in B. mori silk fibroin: Multinuclear NMR Study of the model peptide (Ala-Gly)) defines a heterogeneous antiparallel antipolar mode of assembly in the Silk II form. Macromolecules 2015, 48, 28-36. [CrossRef]

34. O'Sullivan, A.C. Cellulose: The structure slowly unravels. Cellulose 1997, 4, 173-207. [CrossRef] 
35. VanderHart, D.L.; Atalla, R.H. Studies of microstructure in native celluloses using solid-state carbon-13 NMR. Macromolecules 1984, 17, 1465-1472. [CrossRef]

36. Sugiyama, J.; Vuong, R.; Chanzy, H. Electron diffraction study on the two crystalline phases occurring in native cellulose from an algal cell wall. Macromolecules 1991, 24, 4168-4175. [CrossRef]

37. Habibi, Y.; Lucia, L.A.; Rojas, O.J. Cellulose nanocrystals: Chemistry, self-assembly, and applications. Chem. Rev. 2010, 110, 3479-3500. [CrossRef]

38. Goldberg, R.N.; Schliesser, J.; Mittal, A.; Decker, S.R.; Santos, A.F.; LO, M.; Freitas, V.L.S.; Urbas, A.; Lang, B.E.; Heiss, C.; et al. A thermodynamic investigation of the cellulose allomorphs: Cellulose(am), cellulose I $\beta(\mathrm{cr})$, cellulose II(cr), and cellulose III(cr). J. Chem. Thermodyn. 2015, 81, 184-226. [CrossRef]

39. Sorieul, M.; Dickson, A.; Hill, J.; Pearson, H. Plant Fibre: Molecular Structure and Biomechanical Properties, of a Complex Living Material, Influencing Its Deconstruction towards a Biobased Composite. Materials 2016, 9, 618. [CrossRef]

40. Poma, A.B.; Chwastyk, M.; Cieplak, M. Coarse-grained model of the native cellulose I $\alpha$ and the transformation pathways to the $\mathrm{I} \beta$ allomorph. Cellulose 2016, 23, 1573-1591. [CrossRef]

41. Brett, C.T. Cellulose microfibrils in plants: Biosynthesis, deposition, and integration into the cell wall. In International Review of Cytology; Academic Press: Cambridge, MA, USA, 2000; Volume 199, pp. 161-199.

42. Anderson, C.T.; Carroll, A.; Akhmetova, L.; Somerville, C. Real-time imaging of cellulose reorientation during cell wall expansion in Arabidopsis roots. Plant Physiol. 2010, 152, 787-796. [CrossRef]

43. Newman, R.H.; Hemmingson, J.A. Carbon-13 NMR distinction between categories of molecular order and disorder in cellulose. Cellulose 1994, 2, 95-110. [CrossRef]

44. Terzi, A.; Storelli, E.; Bettini, S.; Sibillano, T.; Altamura, D.; Salvatore, L.; Madaghiele, M.; Romano, A.; Siliqi, D.; Ladisa, M.; et al. Effect of processing on structural, mechanical and biological properties of collagen-based substrates for regenerative medicine. Sci. Rep. 2018, 8, 1429. [CrossRef] [PubMed]

45. Terzi, A.; Gallo, N.; Bettini, S.; Sibillano, T.; Altamura, D.; Campa, L.; Natali, M.L.; Salvatore, L.; Madaghiele, M.; De Caro, L.; et al. Investigation of processing-induced structural changes in horse type-I collagen at sub and supramolecular levels. Front. Bioeng. Biotechnol. 2019, 7, 203. [CrossRef] [PubMed]

46. De Caro, L.; Giannini, C.; Lassandro, R.; Scattarella, F.; Sibillano, T.; Matricciani, E.; Fanti, G. X-ray dating of ancient linen fabrics. Heritage 2019, 2, 2763-2783. [CrossRef]

47. Altamura, D.; Lassandro, R.; Vittoria, F.A.; De Caro, L.; Siliqi, D.; Ladisa, M.; Giannini, C. X-ray microimaging laboratory (XMI-LAB). J. Appl. Cryst. 2012, 45, 869-873. [CrossRef]

48. Sibillano, T.; De Caro, L.; Altamura, D.; Siliqi, D.; Ramella, M.; Boccafoschi, F.; Ciasca, G.; Campi, G.; Tirinato, L.; Di Fabrizio, E.; et al. An Optimized Table-Top Small-Angle X-ray Scattering Set-up for the Nanoscale Structural Analysis of Soft Matter. Sci. Rep. 2014, 4, 6985. [CrossRef] [PubMed]

49. Rich, A.; Crick, F.H.C. The molecular structure of collagen. J. Mol. Biol. 1961, 3, 483-506. [CrossRef]

50. Okuyama, K.; Xu, X.; Iguchi, M.; Noguchi, K. Revision of collagen molecular structure. Biopolymers 2006, 84, 181-191. [CrossRef]

51. Okuyama, K.; Hongo, C.; Wu, G.; Mizuno, K.; Noguchi, K.; Ebisuzaki, S.; Tanaka, Y.; Nishino, N.; Bachinger, H.P. High-resolution structures of collagen-like peptides [(Pro-Pro-Gly)(4)-Xaa-Yaa-Gly-(Pro-Pro-Gly)(4)]: Implications for triple-helix hydration and Hyp $(X)$ puckering. Biopolymers 2009, 91, 361-372. [CrossRef]

52. Bella, J.; Brodsky, B.; Berman, H.B. Hydration structure of a collagen peptide. Structure 1995, 3, 893-906. [CrossRef]

53. Giraud-Guille, M.-M. Liquid crystallinity in condensed type I collagen solutions: A clue to the packing of collagen in extracellular matrices. J. Mol. Biol 1992, 224, 861-873. [CrossRef]

54. Fossey, S.A.; Némethy, G.; Gibson, K.D.; Scheraga, H.A. Conformational energy studies of beta-sheets of model silk fibroin peptides. I. Sheets of poly(Ala-Gly) chains. Biopolymers 1991, 31, 1529-1541. [CrossRef] [PubMed]

55. Guo, C.; Zhang, J.; Jordan, J.S.; Wang, X.; Henning, R.W.; Yarger, J.L. Structural Comparison of Various Silkworm Silks: An Insight into the Structure-Property Relationship. Biomacromolecules 2018, 19, 906-917. [CrossRef]

56. Zhou, C.Z.; Confalonieri, F.; Jacquet, M.; Perasso, R.; Li, Z.G.; Janin, J. Silk Fibroin: Structural Implications of a Remarkable Amino Acid Sequence. Proteins 2001, 44, 119-122. [CrossRef] [PubMed] 
57. Leppänen, K.; Andersson, S.; Torkkeli, M.; Knaapila, M.; Kotelnikova, N.; Serimaa, R. Structure of cellulose and microcrystalline cellulose from various wood species, cotton and flax studied by X-ray scattering. Cellulose 2009, 16, 999-1015. [CrossRef]

58. Mao, Y.; Su, Y.; Hsiao, B.S. Probing structure and orientation in polymers using synchrotron small- and wide-angle X-ray scattering techniques. Eur. Polym. J. 2016, 81, 433-446. [CrossRef]

59. Diaferia, C.; Sibillano, T.; Balasco, N.; Giannini, C.; Roviello, V.; Vitagliano, L.; Morelli, G.; Accardo, A. Hierarchical Analysis of Self-Assembled PEGylated Hexaphenylalanine Photoluminescent Nanostructures. Chem. Eur. J. 2016, 22, 16586-16597. [CrossRef]

60. Diaferia, C.; Sibillano, T.; Altamura, D.; Roviello, V.; Vitagliano, L.; Giannini, C.; Morelli, G.; Accardo, A. Structural Characterization of PEGylated Hexaphenylalanine Nanostructures Exhibiting Green Photoluminescence Emission. Chem. Eur. J. 2017, 56, 14039-14048. [CrossRef]

61. Diaferia, C.; Gianolio, E.; Sibillano, T.; Mercurio, F.A.; Leone, M.; Giannini, C.; Balasco, N.; Vitagliano, L.; Morelli, G.; Accardo, A. Cross-beta nanostructures based on dinaphthylalanine Gd-conjugates loaded with doxorubicin. Sci. Rep. 2017, 7, 307. [CrossRef]

(C) 2020 by the authors. Licensee MDPI, Basel, Switzerland. This article is an open access article distributed under the terms and conditions of the Creative Commons Attribution (CC BY) license (http://creativecommons.org/licenses/by/4.0/). 